\title{
Armenozid - Genozid, Gruppen-, Kollektiv- und Völkermord(en)
}

\author{
Literaturbericht zur vergleichenden Völkermordforschung (Teil I)
}

»By sgenocider we mean the destruction of a nation or of an ethnic group. This new word, coined by the author to denote an old practice in its modern development, is made from the ancient Greek word genos (race, tribe) and the Latin cide (killing), thus corresponding in its formation to such words as tyrannicide, homocide, infanticide, etc. Generally speaking, genocide does not necessarily mean the immediate destruction of a nation, except when accomplished by mass killings of all members of a nation. It is intended rather to signify a coordinated plan of different actions aiming at the destruction of essential foundations of the life of national groups, with the aim of annibilating the groups themselves. The objectives of such a plan would be disintegration of the political and social institutions, of culture, language, national feelings, religion, and the economic existence of national groups, and the destruction of the personal security, liberty, health, dignity, and even the lives of the individuals belonging to such groups. Genocide is directed against the national group as an entity, and the actions involved are directed against individuals, not in their individual capacity, but as members of the national group. " ${ }^{1}$

\section{Armenozidale Erinnerungskultur}

Der Band mit "Stimmen aus Deutschland $\aleph^{2}$ ist vor allem, aber nicht nur, als Gedenkjahrband Beitrag und Textsammlung zur armenozidalen Erinnerungskultur. Er soll an das Destruktionsereignis im Osmanischen Staat während des Ersten Weltkriegs erinnern, das im armenischen Selbstverständnis »Medz Aghed « (die große Katastrophe) heißt und in der internationalen sozial-) wissenschaftlichen Forschung Genocide oder Armenocide genannt wird. Ich nenne, wie im Buch »Völkermord(en). Genozidpolitik im 20. Jahrhunderts « ${ }^{3}$ eingehend begründet, »den Armeniermord, also die "Ausrottung " oder »Vernichtung« der Armenier im Osmani-

1 Raphael Lemkin, Axis Rule in Occupied Europe, Carnegy Endowment for International Peace Division of International Law 1944, S. 79.

2 Ischchan Tschiftdschjan (Hg.), Zum 90. Gedenkjahr des Völkermordes an den Armeniern 1915-2005. Stimmen aus Deutschland. Antworten, Aufsätze, Essays, Reden, armenische Angenzengenberichte, Leipzig 2005.

3 Aachen 2006.

ZfP 55. Jg. 4/2008 
schen Staat während des Ersten Weltkriegs, in Anlehnung an ein gebräuchliches analoges Kunstwort Armenozid und bilde das Adjektiv armenozidal entsprechend.

Der Herausgeber, Ischchan Tschiftsdchjan (*1974), ein im Libanon geborener armenischer Doktorand an der Karl-Marx-Universität Leipzig, hat das Buch zum »Holocaust before the Holocaust « (so der Friedensnobelpreisträger Elie Wiesel 1986) anregend durchkomponiert: Dem Geleitwort des armenischen Katholikos von Kilikien folgt eine editorische Einleitung zum Zusammenhang vom Erinnern und Anerkennen des Völkermords an den Armeniern im Osmanischen Staat während des Ersten Weltkriegs, auch als Gerechtigkeitsmoment - wobei hier, wie auch in den meisten Beiträgen, deutlich wird, dass die im Frühjahr 2005 gestartete und schon bald erfolgreiche Initiative von CDU-Bundestagsabgeordneten um Christoph Berger und Katherina Reiche zur Anerkennung des Armenozids durch den Deutschen Bundestag im Juni 2005 auch für viele »Armenierfreunde« überraschend kam.

Die drei Hauptrubriken des Bandes sind etwa drei Dutzend »Antworten « auf ein halbes Dutzend gestellter Fragen zum »Armenischen Völkermord «, sechs kurze wissenschaftliche »Aufsätze« und neun »Essays«. Diesen folgen drei weitere Rubriken: zwei thematisch bezogene »Reden «, sechs historische »Augenzeugenberichte« und ein dokumentarischer »Anhang « mit Erklärungen und Resolutionen zum weiter wissenschaftlich aufzuarbeitenden, moralisch zu erinnernden und (vor allem vom türkischen als Rechtsnachfolger des Osmanischen Staats) politisch anzuerkennenden Völkermord an den Armeniern, der leider sowohl im editorischen Fragenset als auch in (wenigen) weiteren Beiträgen wie als schlechte Übersetzung des USamerikanischen »Armenian Genocide«, nämlich fälschlich »Armenischer Völkermord « anstatt zutreffend »Völkermord an den Armeniern« genannt wird. Ähnlich verwirrt wirkt die im Band abgedruckte Intellektuellen-Resolution des deutschen PEN-Zentrums vom 25. Mai 2005 mit ihrer Aufforderung an »die türkische Öffentlichkeit« - nicht aber an die Regierung der Türkischen Republik -, »endlich den Völkermord an den Armeniern« als solchen anzuerkennen.

Die meisten "Antworten « kommen von sich oft auf Johannes Lepsius (18581926) beziehenden evangelischen Pastoren, dazu einige von prominenten Zeithistorikern und (weniger) bekannten Schriftstellern. Deutlich wird unter erinnerungskulturellen Aspekten die über Lesergenerationen andauernde Wirksamkeit von Franz Werfels zuerst 1933 veröffentlichtem großen Armemierroman Die vierzig Tage des Musa Dagh, an den in gewisser Weise auch Edgar Hilsenraths bedeutender »historischer Roman aus dem Kaukasus « Das Märchen vom letzten Gedanken anzuschließen versuchte. Aber auch hier, und später im gesonderten Beitrag von Dr. Adelheid Latchinian, wird ans mutige lebenslange Engagement Armin T. Wegners (1896-1978) und dessen dokumentarische Fotos des »Armeniermords «1915/16 erinnert.

Für die wissenschaftliche Aufarbeitungen des Armenizod wichtig sind vor allem die »Aufsätze« des Bandes. Zu diesen bietet der subjektbezogene Essay von Tessa Hofmann (»Den stimmlos Gemachten eine Stimme geben«) einen respektablen $\mathrm{Zu}-$ gang durch den Aufruf zur Unterstützung der Forderung von Armenozidüberle- 
benden und ihrer Angehörigen »nach Anerkennung des Verbrechens durch den Gesetzgeber und die Regierung des Landes, das das Verbrechen begangen hat « und das gesellschaftlich »durch die Gemoziderfahrung objektiv belastet ist « - auch wenn dies alles »bis zum heutigen Tag von der türkischen Regierung namens der Tätergesellschaft geleugnet « wird (so zusammenfassend und zutreffend Wolfgang Benz in seinem Essaytext).

Ans »Tabu « - auch »als Basis paranoider Phantasmen« - der Benennung des Armenozid als Genozid und entsprechender Verfolgung in der gegenwärtigen Türkei erinnert aus psychoanalytischer Sicht Ester Schulz-Goldstein. Der Hamburger Jurist und Rechtsprofessor Otto Luchterhandt, der auch schon im Juni 2003 über den »türkisch-armenischen Konflikt, die Deutschen und Europa « gutachtete ${ }^{4}$, publizierte sein für den Deutschen Bundestag erarbeitetes »Memo « als Aufsatz in Langfassung. So zutreffend des Autors fallbezogene juristische Wertung des objektiven und subjektiven Völkerrechtsstaftatbestand - Armenozid als Völkermord - auch ist, so unangemenssen wirkt Luchterhandts totalitärbürokratisches Diktum: »Nur ein Jurist, sei er Richter oder Rechtsgelehrter, vermag die Frage, ob bestimmte Geschehnisse, Taten oder Tatsachen als Völkermord zu qualifizieren sind, fachgerecht zu beantworten.«

Hier hätte der Rechtswissenschaftler Luchterhandt, der Ralf Dahrendorfs freundliche Kritik an den deutschen "Juristen des Monopols « ${ }^{5}$ einfach beiseite schiebt, besser seiner "Pflicht des Tages « (Goethe) nachgehen und nachvollziehen sollen, was zwei Aufsätze und zwei Dutzend Seiten weiter Georg Meggle mit Bezug auf eigene Vorüberlegungen zu Kollektividentitäten ${ }^{6}$ zur Logik der UN-Völkermorddefinition grundlegend entwickelt, unter welche dieser Autor sowohl den Holocaust als auch den Armenozid (im Wissen, dass die angemessene Bezeichnung beider Kollektivmord wäre) fasst und was sich aus Meggles kritisch-systematischem Zugang zum historischen Völkermord an den Armeniern während des Ersten Weltkriegs im Osmanischen Staat für Täter und Opfer und ihre und deren Kinder nach mehr als drei Generationen, aber auch mit Blick auf Deutschlands »Sonderrolle« konkret ergibt. Nämlich etwa die historische und aktuelle Wahrheitspflicht, die auch kein Verschweigen einiger » Massaker von Seiten der Armenier an den Türken" duldet, oder die Angst heutiger türkischer Intellektueller, den »Völkermord an den Armeniern furchtlos beim Namen « zu nennen, oder Meggles differenzierte Bewertung der leider nicht »expliziten «, sondern nur »impliziten « einstimmigen Anerkennung des Völkermords an den Armeniern durch den Deutschen Bundestag am 15. Juni 2005 als einen »ungeheuren Fortschritt«.

Als ähnlich kundig und engagiert sind die »Anregungen zu einem kritischen Tria$\log$ « des Bielefelder Zeitgeschichtlers Hans-Walter Schmuhls zu bewerten. Diese begründet er mit seiner These der spezifischen Modernität des Völkermords an den

4 http://www.deutsch-armenische-gesellschaft.de/dag/rgenlu1.pdf (abgerufen am 30.6.2008).

5 Gesellschaft und Demokratie in Deutschland, München 1965, S. 260-276.

6 http://www.unileipzig.de/ philos/meggle/\&publikationen/2005f.pdf (abgerufen am 30.6. 2008) 
Armeniern als Ausdruck des Kernkonflikts »zweier neu entstehender, in Konkurrenz zueinander stehender Nationalismen " von Armeniern und Türken in Reaktion »auf die imperialistische Politik der europäischen Mächte mit der Herausbildung einer nationalen Identität« entsprechend »der europäischen Idee des ethnisch homogenen Nationalstaates«. Auch in diesem Ideologietransfer liege »die Mitschuld Europas an der Vernichtung des armenischen Bevölkerungsteils des Osmanischen Reiches«. Darüber hinaus zieht Schmuhl auch Verbindungslinien vom Völkermord an den Armeniern zu den nationalsozialistischen Genoziden an Juden, Slawen, Sinti und Roma. Im Vergleich mit »vormodernen « Gewalt-, Kollektivmord- und Vernichtungsaktionen, etwa auch während des Zweiten Weltkriegs in Bosnien durch Tschetniks ${ }^{7}$, wird Schmuhls Hinweis auch empirisch plausibel, weil bei diesen Massakern von »rohen Kräften«, auch im Vergleich mit dem beim Armenozid planvollstaatskriminellen Vernichtungshandeln, alle infrastrukturellen Voraussetzungen für Völkermord/Genozid als besonderes (Staats-)Verbrechen fehlten. Gut, dass HansWalter Schmuhl es nicht bei dieser Skizze belassen hat, sondern parallel eine am »sozialen Handeln « Max Webers geschulte, sinnbezogene Genoziddefinition als über Fallstudien hinausgehenden Beitrag zur allgemeinen sozialwissenschaftlichen Völkermorddiskussion publiziert hat ${ }^{8}$.

Auf neun Seiten fasst der Wirtschaftshistoriker Hilmar Kaiser seine eigenen Forschungsergebnisse zum Armenozid zusammen (der leider auch von ihm statt »Völkermord an den Armeniern« als »Armenischer Völkermord « bezeichnet wird). Der Autor betont zutreffend, dass es angesichts der Phalanx von Leugnern zunächst darum ging, diesen »Völkermord zu beweisen « und dass diese Hauptarbeit andere, vor allem wirtschaftliche, »Aspekte des Völkermords « vernachlässigte, $z$. B. die von ihm selbst inzwischen dokumentierte lebensprotektiv-armenierschützende und teilweise lebensrettende »Haltung der Deutschen Bank zum Armenischen Völkermord «. Auch für Kaiser steht die Modernität dieses Völkermords außer Frage, sollte doch »die Revolution der osmanischen Gesellschaft auf Grundlage einer nationalistisch-türkischen Ideologie« erfolgen und die historisch »komplexe osmanische Gesellschaft [...] einer `türkischen< Platz machen «. Im Ausblick erinnert Kaiser an die Erfordernis und seinen eigenen Forschungsanspruch, »neben der Vertiefung einzelner Aspekte des Völkermords das Verbrechen in größere Kontinuitätslinien « und dabei besonders »in die Kontinuitätslinien der osmanischen Gesellschaft« einzuordnen.

Als Mitglied der Arbeitsgruppe für Genozidforschung an der Universität Zürich hatte Dominik J. Schaller schon 2001/02 eine vergleichende Perspektive in der Völkermordforschung versucht ${ }^{9}$. Hier schließt sein Beitrag an. Zugleich bemüht sich

7 Tomislav Dulic, Utopias of Nation. Local Mass Killing in Bosnia and Herzegovina, 1941-42, Uppsala 2005 [ = Studia Historica Upsaliensia].

8 »Der Völkermord an den Armeniern 1915-1917 in vergleichender Perspektive « in: Fikret Adanir/Bernd Bonwetsch (Hg.), Osmanismus, Nationalismus und der Kaukasus. Muslime und Christen, Türken und Armenier im 19. und 20. Jabrbundert,. Wiesbaden 2005 [= Kaukasienstudien/Caucasian Studies 9], S. 271-299.

9 http://www.hist.net/kieser/aghet/Essays/EssaySchaller.html (abgerufen am 30.6.2008). 
der Autor in seiner knappen Forschungsskizze aber auch, über den wissenschaftlichen »Vergleich, der sich als bedeutende Methode der Genozidforschung bewährt hat [zum] Feststellen von Gemeinsamkeiten und Unterschieden « im Mordhandeln hinauszugehen und im Anschluss an ein unveröffentlichtes Buchmanuskript von Raphael Lemkin (1900-1959) »Kontinuitätslinien vom Völkermord an den Armeniern und von kolonialen Genoziden - wie beispielsweise demjenigen an den Herero und Nama in der ehemaligen deutschen Kolonie Südwestafrika - zum nationalsozialistischen Judenmord aufzuzeigen «. Dabei besteht für Schaller »kein Zweifel daran, dass die erfolgreiche Transformation des osmanischen Vielvölkerreichs in einen türkischen Nationalstaat, die der Ermordung und Vertreibung von Armeniern und Griechen vorausgegangen war, die Nationalsozialisten nachhaltig beeindruckt hatte.«

Die Texte von Hofmann, Kaiser, Schulz-Goldstein, Schaller und insbesondere von Schmuhl und Meggle im Gedenksamelband halte ich für ambitionierte Beiträge, die über den anlassstiftenden 90. Jahrestag des formellen Armenozid-Beginns durch Festnahme armenischer Honoratioren in Constantinopel am 24. April 1915 hinausgehen und auch im Sinne vergleichender Völkermordforschung(en) zur Genozidpolitik des/im 20. Jahrhundert/s anregen - wobei gelegentlich das der heutigen Entwicklung auf dem Balkan (und seiner internationalen Gerichtsbarkeit in Den Haag) seit etwa fünfzehn Jahren zur Deutung unterliegende aktuelle Leitkonzept der ethnisch-demographischen Homogenisierung (»ethnic cleansing «) retrospektivisiert, also auf das erste crimen magnum des vergangenen Jahrhunderts, den Armenozid, umstandslos rückbezogen und insofern verkürzt angewandt wird. Völkermorde/ Genozide lassen sich jedoch nicht im Allgemeinen ethnischer »Säuberungs-« oder »demographischer Homogenisierungsaktionen« auflösen.

Liest man die genannten sechs Autoren, Thesen und Forschungsskizzen im $\mathrm{Zu}-$ sammenhang mit Publikationen internationaler Völkermordforscher der mittleren Wissenschaftlergeneration, die sich um die Fachzeitschrift Journal of Genocide Research (seit 1998), um den komparatistisch angelegten Sammelband des von HansLukas Kieser und Dominik Schaller an der Universität Zürich begründeten Forschungs- und Publikationszusammenhangs zum »Völkermord an den Armeniern und die Shoah $\ll^{10}$ und um das Anfang 2005 gegründete »European Network of Genocide Scholars « (ENoGS) gruppieren, und vergleicht sie etwa mit Diskussionsbeiträgen von Mark Lewine ${ }^{11}$, Martin Shaw ${ }^{12}$ oder Ronald Bloxham ${ }^{13}-$ dann wird deutlich, dass es inzwischen relevante, über »Fallstudien« hinausgehende gesicherte

10 Hans-Lukas Kieser, Dominik J. Schaller (Hg.), Der Völkermord an den Armeniern und die Shoah, Zürich 2002.

11 http://www.learner.org/channel/courses/worldhistory/support/reading_22_2.pdf (abgerufen am 30.6.2008, »Why Is the Twentieth Century the Century of Genocide ?« in: Journal of World History 11 [2000] 2, S. 305-336; deutsch in: Zeitschrift für Weltgeschichte [ZWG], 11 [2004] 2: 9-37).

12 Martin Shaw, War E Genocide. Organized Killing in a Modern Society, Oxford 2003.

13 The Great Game of Genocide. Imperialism, Nationalism, and the Destruction of the Ottoman Armenians, Oxford, 2005. 
(sozial-) wissenschaftliche Erkenntnisse zum Genozid und zur Völkermordpraxis, zu genozidalen Absichten, Handlungen und ihren Folgen gibt, die im Sinne aktivvergemeinschafteten Wissens und nicht-ideologischen Gedächtnisses (»una memoria histórica, testimonial « [Jorge Semprún]) theoretisch irreversibel und insofern auch praktisch nur gewaltsam als ideeller Zivilisationsbruch rücknehmbar sind.

\section{II. »Vertreibung«: »Umsiedlung« als Symptom}

Der Band Völkermord oder Umsiedlung?14 der beiden Hamburger Zeitgeschichtler Jörg Berlin und Adrian Klenner ist nach den von mir in Völkermord(en). Genozidpolitik im 20. Jabrbundert ${ }^{15}$ zusammenfassend vorgestellten Büchern zum Armenozid von Vahakn N. Dadrian (1996/19972) zur reichsdeutschen Mitverantwortung, Taner Akcam (1996/2004²) zu den Istanbuler Nachkriegsprozessen 1919 und zu Wolfgang Gusts (2005) Neuherausgabe von Völkermorddokumenten aus dem Auswärtigen Amt 1915/16 die letzterschienene "große« dokumentarische Darstellung zum Armenozid. Das Buch enthält nach einer Problemeinführung einen historischen Überblick zum »Schicksal der Armenier im Osmanischen Reich «. Dieser überzeugt als »Darstellung « ebenso wenig wie der gesamte Band mit seinen insgesamt 259 Dokumenten auf gut 300 Seiten. Erstens zeigt die etwa fünfzigseitige Überblicksdarstellung der Autoren, dass weder beim Armenozid im speziellen noch beim Genozid im allgemeinen ein zeitgeschichtliches Gutgemeint ausreicht. Zweitens verdeutlichen die nicht immer aus Primärquellen, Dokumenteneditionen, Erstpublikationen edierten zahlreichen, in sechs historischen Kapiteln gruppierten Dokumente, dass jede noch so gut gemeinte Dokumentenreihung bestenfalls Facetten veranschaulichen, aber kein Gesamtbild ergeben kann. Drittens finden sich im Dokumententeil (zu) oft Texte, die (unüberprüft) nicht aus Primärquellen übernommen wurden, sondern aus Reprints, Nachdrucken und Sonderausgaben. So etwa Dokument 237: Die zuerst in den USA 1921 erschienenen Posthumous Memoirs of Talaat Pasha werden nicht nach der Primärquelle Current History zitiert, wobei der Zeitschriftenjahrgang unter anderem in der Hamburger ZB Recht unter der Signatur IIA Z 106 durchaus greifbar wäre. Ein weiteres Beispiel bietet Dokument 73: Die Verordnung zur »Zwangsumsiedlung « vom 27. Mai 1915 (die im Buch als »Gesetz« erscheint und zuerst 1917 in Constantinopel veröffentlicht und so auch in die Lepsius-Aktenedition von 1919 als Dokument 71 übernommen wurde), wird sowohl in einer gekürzten Version als auch aus einem englischen Buch und dazu noch falsch rückübersetzt präsentiert: Im entscheidenden Artikel 2 der »Notverordnung " war nämlich die Rede von "déplacer et installer dans d'autres localités, séparément ou conjoinement «. Das aber meint nicht, wie im Buchdokument 73 behauptet, Personen »einzeln oder insgesamt fortschaffen oder an anderen Orten ansiedeln«, sondern sie »in Bewegung zu setzen und einzeln oder gemeinsam an-

14 Jörg Berlin und Adrian Klenner (Hg.) Völkermord oder Umsiedlung ? Die Armenier im Osmanischen Reich - Darstellung und Dokumente, Köln 2006.

15 Aachen 2006, S. 113-116. 
dernorts unterzubringen «. Desweiteren ist die hier dominierende historiographische Sicht keine historische Perspektive, weil im Grunde auf deutsche Geschichte (mit gelegentlicher Parallelsicht auf türkische) bezogen und damit national angelegt. Auch fehlt jede »Anstrengung des Begriffs « (G.W.F. Hegel), etwa in Form hier besonders dringlich erforderlicher Begriffsbestimmung und -differenzierung bei Völkermord [und] Genozid einerseits vs. Umsiedlung [und] Deportation andererseits, weshalb die Autoren in ihrem »historischen Überblick « auch meinen feststellen zu können, dass bei aller Objektivität und Dichte so vieler zeitgenössischer »von einander unabhängiger und unvoreingenommener Beobachter und Zeitzeugen [...] das Vorhandensein eines Konzepts für einen Völkermord nicht endgültig « bewiesen wäre. Diese falsche Bewertung entwertet sowohl die hier erneut dokumentierten Berichte vor allem deutscher, österreichischer und US-amerikanischer Augen- und Zeitzeugen als Quellen als auch und insbesondere alle Hinweise des bekannten »Armenierfreunds" Lepsius. Dieser erkannte nämlich bereits 1916 als erster Deutscher, dass das, was zeitgenössisch »Ausrottung « hieß, die »Vernichtung der armenischen Nation" und damit »Völkermord, den die Jungtürken auf dem Gewissen haben«, war $^{16}$. Und auch wenn Lepsius' politische Rolle nach 1919 als Mitherausgeber der großen AA-Edition die eines »objektiven« Apologeten des wilhelminischen Imperialismus gewesen sein mag - als Gerichtssachverständiger sprach Lepsius 1921 öffentlich von der Vernichtung des armenischen Volkes. ${ }^{17}$ Absurd wird es, wenn die Autoren meinen, die am 15. Juni 2005 einstimmig angenommene Resolution des Deutschen Bundestages ${ }^{18}$ wegen der dort nur indirekt erfolgten Kennzeichnung von »Vertreibungen und Massakern an den Armeniern 1915 «als »Völkermord « kritisieren zu sollen - sie selbst aber im Buchtitel den noch stärker verharmlosenden und vor allem von militanten Völkermordleugnern benutzten und im Deutschen ungebräuchlichen Ausdruck »Umsiedlung « verwenden und in ihren eigenen Texten nicht klipp und klar von Völkermord oder Genozid sprechen.

Die Formel »Umsiedlung (türkisch: »tehcir«, auch: »zorunlu göç« oder »sürgün«) - im britischen Englisch als »resettlement« im Doppelsinn von Um- und Wiederansiedlung, im Französischen als »transfert «, »déplacement de population «, auch »transplantation « benützt, wobei Zwangsumsiedlung »le transfert forcé« meint - erinnert nicht nur an die NS-rassistische Wortschöpfung »Umvolkung « faschistischer Ideologen wie Albert Brackmann, Werner Conze und Theodor Schieder, sondern ist, im Gegensatz etwa zu »Vertreibung «, ein im Deutschen höchstungebräuchliches Sprachkonstrukt: Wohl kennen die entsprechenden Bände des "vorbraunen $"$ Meyer-Lexikon ${ }^{19}$ Siedlung/en und Siedlungsformen ebenso wie das von Wolfgang Pfeifer und Mitarbeiter/innen erarbeitete »Etymologische Wörterbuch $\ll\left(\operatorname{dtv} 3358,1995^{3}\right)$ des Berliner Zentralinstituts für Sprachwissenschaft die Tä-

16 »Mein Besuch in Konstantinopel Juli/Aug. 1915 « in: Orient. Monatsschrift für die Wiedergeburt des Ostens, Potsdam, 1 (1919), 1/3, S. 21-33.

17 Tessa Hofmann (Hg.), Der Völkermord an den Armeniern vor Gericht. Der Prozeß Talaat Pascha, Göttingen 1980, S. 56-61.

18 BT-Drucksache 5689.

19 Bibliographisches Institut, 7. Auflage 1929/30. 
tigkeit (be- und an-) »siedeln« im Sinne von sesshaft oder ansässig werden, sich niederlassen - $\mathrm{nicht}$ aber »umsiedeln« oder »Umsiedlung«; genauer: auf »Umsiedlung « heben bis heute vor allem (nicht nur türkische) Armenozidleugner $\mathrm{ab}$, indem auf die entsprechende "Notverordnung " des Osmanischen Staats (27.5.1915) verwiesen wird: »Die Umsiedlung war [...] die aus Sicherheitsgründen vorgenommene Zwangsansiedlung einer bestimmten Gruppe [...] in bestimmten Gebieten zum Zwecke der Verhinderung schädlicher Aktivitäten «, genauer: die »osmanische Regierung [...] siedelte die in der Nähe der Kampfgebiete lebenden Armenier in weiter südlich gelegene osmanische Gebiete und nach Syrien um $\ll^{20}$. Es ist dies die ungebrochene Rechtfertigung von sei es »Umsiedlung «, sei es "Zwangsansiedlung " genannter und 1915 begonnener Ausrottungs-, Vernichtungs- und Völkermordpolitik des Osmanischen Staates, die bereits die jungtürkische Tätergruppe 1916/17 zur Rechtfertigung ihrer »Maßnahme « verbreiten ließ. Die entsprechenden Broschüren erschienen in der damaligen Diplomatensprache französisch zuerst (die erste am 1. März 1916) als »Vérité sur le Movement Révolutionnaire arménien et les mesures gouvernementales/Journal de guerre [...]/Notes d'un officier superieur russe $[\ldots] \ll^{21}$ und später als $\gg$ Aspirations et agissement révolutionnaires des Comité Arméniens avant et après la proclamation de la Constitution Ottomane «22... wer auch immer nachhaltig recherchiert kann diese Genozidapologien in öffentlichen Bibliotheken leicht auffinden. Und wer sich darüber hinaus noch die Mühe macht, fünf Jahrgänge von Schulthess' Europäischer Geschichtskalender ${ }^{23}$ als zeitgenössische Quelle durchzusehen, wird v o $\mathrm{r}$ der osmantürkischen Regierungspolemik vom 6. Juni 1915 als Antwort auf die Erklärung der Triple-Ententemächte [vom 24. Mai 1915], nämlich die Regierungsverantwortlichen für diese neuen Verbrechen gegen Menschheit und Zivilisation »in Person verantwortlich zu machen $\ll^{24}$, $\mathrm{k}$ e i n e Hinweise auf irgendwelche armenischen Verschwörungen oder Aufstandspläne gegen das jungtürkische Regime finden. Was Armenozidrechtfertigungen betrifft, so war dies ein ideologisches Operationsfeld ehemaliger prominenter Wissenschaftler und faschistischer Ideologen des »Dritten Reiches « während des Zweiten Weltkriegs, etwa von Carl Mühlmann, Gotthard Jäschke, Franz Ronneberger. Mühlmann beispielsweise übernahm die damalige jungtürkische Propaganda als »Tatsache, dass ein großer Teil des armenischen Volkes durch die >Aussiedlung ¿ zugrunde gegangen ist« und verwies als »tiefere Ursache des türkischen Armenierhasses« auf die türkistisch-turanistische Ideologie mit ihrem rassistischen Überwertigkeitsanspruch $^{25}$.

20 Institut für Außenpolitik (Hg.), Das Armenierproblem in neun Fragen und neun Antworten, Ankara 1982, S. 28-29.

21 Constantinople 1916; 2. Aufl. 1919.

22 Istanbul 1917.

23 N.F., 51 (1910) bis 56 (1915).

24 Ebd., 56 (1915), S. 1151.

25 Carl Mühlmann, Das deutsch-türkische Waffenbündnis im Weltkriege, Leipzig 1940, S. $276 \mathrm{f}$. 
Zusammenfassend verstricken sich auch die Autoren namentlich im Umsiedlungskomplex wie vor ihnen all jene Historiker und Soziologen ${ }^{26}$, die jede »Vernichtungsabsicht « bestreiten, in jene Widersprüche, die Yves Ternon zutreffend so kennzeichnete: »Die Absurdität [...] lässt sich mit folgender Formel wiedergeben: Nichts ist ihnen [den Armeniern] geschehen, aber sie haben es verdient. «27 Im konkreten Armenozid-»Fall «fallen die Autoren noch hinter unser heutiges lexikalisch geronnenes wissenschaftliches Wissen zurück ${ }^{28}$ :

»Der bekannteste schriftliche Genozidbefehl - erhalten als Telegramm - stammt vom türkischen Innenminister Talat Pascha für die Ausrottung der Armenier im Jahre 1915: >Ihnen wurde bereits mitgeteilt, dass die Regierung durch Befehl der Versammlung (Jemiet) beschlossen hat, die in der Türkei lebenden Armenier restlos auszurotten. Diejenigen, die sich diesem Befehl widersetzen, können nicht mehr für die Regierung im Amt bleiben. Ohne Rücksicht auf Frauen, Kinder und Kranke [...] muss ihrer Existenz ein Ende bereitet werden.«

$$
\text { III. »... die Armenierfrage diskutiert ..." }
$$

Das in Istanbul ansässige Türkei-Büro der deutschen Heinrich-Böll-Stiftung veröffentlichte im März 2006 einen Sammelband zur Diskussion »der Armenierfrage « in der Türkei ${ }^{29}$. Die Büroleiterin, Dr. Ulrike Dufner, will wohl »die damaligen tragischen Geschehnisse « und damit die »Geschichte der Armenier in der Türkei « angesprochen haben, »dabei jedoch nicht auf die Ereignisse selbst eingehen«. Ihr Anliegen im speziellen ist es, »Stereotypen mit der Realität [zu] konfrontieren«, um zu irritieren und anzuregen. Es ist gut, dass einige Beiträge in diesem Buch über dieses küchenpädagogische Ansinnen und das ihm unterliegende Klippschulbild von »Stereotypen des >Anderen« « - vermutlich gemeint: ego/alter ego - hinausgehen. Und es ist noch besser, dass das Buch zweisprachig, deutsch und türkisch, erschien.

Der Band nimmt den ironischen Hinweis eines fiktiven Theater-Direktors: »Wer vieles bringt, wird manchem etwas bringen «(Faust I: 97) beim Wort. Die sechs Aufsätze sind recht heteronom-sujetbezogene Darstellungen, Berichte und Hinweise. Alexander Iskandarian (Eriwan) trägt den heutigen staatsarmenischen Standpunkt zum Armenozid und Hinweise auf einige neuere armenische Publikationen vor. Über seine Ausstellung zur Anregung türkischer Erinnerungsarbeit unter dem Titel »Mein lieber Bruder« (Sireli Yeghpajrs) von Januar und Oktober 2005 in Instanbul, Oktober/November in München (und nun auch im April 2006 in Köln) berichtet Autor und Organisator Osman Köker ${ }^{30}$. Diese Beitragsgruppe enthält auch einen

26 Kurt Steinhaus, Soziologie der türkischen Revolution. Zum Problem der Entfaltung der bürgerlichen Gesellschaft in soziökonomisch schwach entwickelten Ländern, Frankfurt/ Main 1969.

27 Yves Ternon, Der verbrecherische Staat. Völkermord im 20. Jabrbundert, Hamburg1996, S. 152.

28 Gunnar Heinsohn, Lexikon der Völkermorde, Reinbek 1998, S. 351.

29 Ulrike Dufner u.a., Wenn man die Armenierfrage diskutiert ... / Ermeni sorunu tartisilirken ..., Istanbul 2006.

30 Siehe auch Osman Köker, Armenier in der Türkei vor 100 Jabren. Mit Postkarten des Sammlers Orlando Carlo Calumeno, Ausstellungskatalog, Istanbul/Köln: 2005/06. 
lokalgeschichtlichen Beitrag. Es geht um die heutige (kurdische) Halbmillionen(groß)stadt in Anatolien, Diyarbekir. Talip Atalay, Kirchenhistoriker und Projektleiter »Diyarbekir Vilayeti'nde Mekteplesme« an dortiger Dicle-Universität, stellt die Stadtzeitung der Jahre 1869-1902 als lokalgeschichtliche Quelle auch für damaliges Leben christlicher Armenier vor. Im Sinn des Vorworts schließlich berichtet einer der Projektleiter einer empirischen Untersuchung über wechselseitige Stereotypen zu »Türkentum « und »Armeniertum « in beiden heutigen Gegenwartsgesellschaften, Ferhat Kentel von der Istanbuler Bilgi-Universität ${ }^{31}$. Dabei zeigte sich, dass einmal das wirkliche wechselseitige Wissen über die jeweils andere Gesellschaft gering ist, dass es zum anderen beiderseits ausgeprägte Negativsteoreotypen gibt, die drittens bei armenischen Befragten hinsichtlich der erwarteten Negativität ihrer Wahrnehmung durch Türken ausgeprägter waren als umgekehrt. Viertens war, wie zu erwarten, der Armenozid als ethnohistorisches Schlüsselereignis der Zeitgeschichte, nach dem ohne »das Ereignis « selbst Völkermord zu nennen speziell gefragt wurde, bei Armeniern ausgeprägter als bei Türken. Fünftens plädiert der Autor für Abbau der Negativstereotypen auf türkischer Seite im Zusammenhang mit fortschreitender Demokratisierung und Pluralisierung der türkischen Gesellschaft.

Der Hauptbeitrag des Bandes umfasst im deutschen Teil etwa 50 Seiten. Der Autor, Hrant Dink (1954-2007), war als Herausgeber der bisher einzigen zweisprachigen armenisch-türkischen Wochenzeitung Agos einer der prominentesten Intellektuellen in der Türkei. Er wurde im Januar2007 beim Verlassen der AgosRedaktionsräume in Istanbul auf offener Straße ermordet, wozu Ralph Giordano treffend erklärte: »Hrant Dink musste sterben, weil er das, was den Armeniern im türkisch-osmanischen Reich 1915/16 widerfuhr, beim Namen nannte: Genozid «32.

In seinem Buchbeitrag hebt Dink bewusst auf seine Doppelidentität als nichttürkischer Armenier und türkischer (Staats-) Bürger ab. Auch Dink, der nach dem Militärputsch 1980 mehrfach inhaftiert wurde, war, wie andere, im EU-Bereich Solidarität erfahrene prominente Autoren, z. B. der Literaturnobelpreisträger Orhan Pamuk 2005 und zuletzt Elif Shafak, die am 21. September 2006 von einem Istanbuler Bezirksgericht freigesprochen wurde, wegen »Beleidigung des Türkentums « nach $\mathbb{3} 301$ Strafgesetzbuch der Türkischen Republik öffentlich angeklagt, erhielt aber zunächst »nur « eine Bewährungsstrafe in der Türkei und im Mai 2006 in Deutschland für sein nachhaltiges und langdauerndes Engagement für Pressefreiheit in der Türkei den Henri-Nannen-Preis.

In seinem Buchbeitrag führt Dink seine vermittelnde Position eines »dritten Weges « zwischen Anerkennung und Leugnung des Armenozid breit aus. Deutlich wird, dass Dink weniger »links vom Möglichen überhaupt « (Walter Benjamin) steht als vielmehr zwischen allen schon besetzten Stühlen Platz zu nehmen versucht. Beide Hauptlager strukturiert der Autor, der »die Armenierfrage« nutzen möchte, um die gegenwärtige türkische Gesellschaft unter dem Primat der weiteren Entwicklung zivilgesellschaftlicher Strukturen im »westlichen« Sinn zu demokratisieren, zu

31 »Armenian \& Turkish Citizens' Mutual Perceptions \& Dialogue« http:// www.tesev.org.tr/etkinlik/ermeni_turk_diyalog.php (aufgerufen 2007).

32 Frankfurter Allgemeine Zeitung vom 27. 01. 2007, S. 39. 
pluralisieren und zu liberalisieren, in Triaden: Die heute aus etwa acht Millionen Menschen bestehende »armenische Welt« lebt im seit September 1991 unabhängigen kaukasischen Kleinstaat (etwa drei Millionen), in der von Dink »Anatolien in der Welt« genannten armenischen »Diaspora (gut 5 Millionen) und besteht auch noch aus einer kleinen, in der Türkei lebenden armenischen Minderheit, der Dink selbst angehört. Insbesondere Diaspora-Armenier hätten sich, seit zwei Jahrzehnten zunehmend erfolgreich, einerseits für die weltweite »Anerkennung der Wahrheit« des Armenozids eingesetzt; aber andererseits zugleich ein Negativstereotyp gegenüber Türken und dem türkischen Staat ausgebildet. Die türkische Seite bestünde aus dem autoritären Staat (soweit mir bekannt in der »Armenierfrage « heute repräsentiert durch harte Leugner wie den Vorsitzenden der staatlichen Gesellschaft für Türkische Geschichte, Yusuf Halacoglu), der Politik (analog verkörpert durch den Vorsitzenden der Arbeiterpartei der Türkei, Dogu Perincek), und der Zivilgesellschaft (analog vertreten durch Rechtsanwalt Kemal Kerincsik, der die Autorin Elif Shafak wegen »Verunglimpfung des Türkentums « anzeigte). Dabei, so Dinks Leitthese zur paradoxen Lage und abgesehen von seinen Hinweisen zum schwierigen Verhältnis der Staaten Türkei und Armenien, blockierten sich die Hauptantipoden (»Diaspora und der türkische Staat) wechselseitig. Diese doppelte Blockade sei unterhalb der Völkermord-Ja-Nein-Ebene aufzulösen, wozu der Autor als »ein Armenier aus der Türkei« beitragen will - und dadurch antidemokratischen Armenozid-Leugnern als »Armenierknecht « gilt. Dinks engagiertes Plädoyer argumentiert mit deutlichem Blick auf die türkische Gegenwartsgesellschaft und die von ihr zivilgesellschaftlich zu entwickelnde Toleranz, Pluralität und Demokratisierung. Diese Sicht hat freilich Folgen: Erstens wird der Blick verengt, was besonders deutlich am Verhältnis des Autors zu Geschichte und Geschichtsschreibung wird. So sehr Dink zum einen und zutreffend positiv bewertet, dass die zunächst staatlich untersagte Konferenz »Die Armenier in der Phase des Niedergangs des Imperiums « schließlich doch im September 2005 an der Istanbuler Bilgi-Privatuniversität stattfinden konn$\mathrm{te}^{33}$ - so zeigt sein Rückbezug auf eine emotional aufgeladene, auch noch die gegenwärtige türkische Mehrheitsgesellschaft auszeichnende Geschichtsschreibung wie sehr auch dieser progressive Intellektuelle (in) der gegenwärtigen Türkei immer noch ins kemalistisch-totalitäre Denken verstrickt ist. Es war Mustafa Kemal, der spätere Atatürk, der zur Gründung der Gesellschaft für Türkischen Geschichte 1931 schrieb: »Geschichte schreiben ist genauso wichtig wie Geschichte machen «34. Diese Atatürk-Formel mit einer Fundstelle [1939] findet sich auf einer Netzseite der türkischen Regierung 35 : Tarih yazmak, tarih yapmak kadar mühimdir. Yazan, ya-

33 Zur Erinnerung: 1982 durften armenische Wissenschaftler zu einer Genozidkonferenz in Israel nicht einreisen, nachdem die damalige türkische Militärregierung Druck ausgeübt hatte. Vgl. dazu Israel W. Charny (Hg.), Toward the Understanding E Prevention of Genocide, Boulder (Colorado) 1984.

34 »Tarih yazmak, tarih yapmak kadar önemlidir.«

35 http://www.kultur.gov.tr/TR/Yonlendir.aspx?F6E10F8892433CFF8FE9074FF19B0005907A48226BB53664, (abgerufen am 30.6.2008). 
pana sadi k kalmazsa, deg is meyen hakikat, insanli g is as i rtacak bir mahiyet alir«, deutsch etwa: »Geschichte schreiben ist genauso wichtig wie Geschichte machen. Der Geschichtsschreiber muss aber treu und loyal sein, ohne diese Eigenschaften wird die Geschichte für den Menschen immer zweifelhaft bleiben.« Zweitens trägt Dink auch in sich Widersprüchliches vor: etwa wenn er einerseits »die weltweiten Bestrebungen der ArmenierInnen« zur Armenozidanerkennung als den innertürkischen Demokratisierungsprozess fördernd, andererseits eher als Beitrag »zur $\mathrm{Zu}$ nahme bestehender Spannungen« wertet. Drittens behandelt der Autor manches zu oberflächlich: Wenn z. B. das türkische Verfassungsgericht das Verbreitungsverbot eines Genozid-Buchs von Vahakn N. Dadrian mit der zynischen Begründung aufhob, »es gäbe nicht mehr viele ArmenierInnen im Land, die man durch das Buch aufhetzen könnte «, kann nicht nur resultathaft das Urteil interessieren, sondern müsste auch die »Begründung « kritisch kommentiert werden. Viertens wirkt Dinks Plädoyer für »den Willen «, der seiner Meinung nach ausreichte, aber beiden Seiten fehlte, historisch naiv und politisch hilflos. Fünftens, und insofern erscheint angemessen, dass der Autor sich nicht mit der Bedeutung des Armenozids selbst beschäftigt, ist Dinks Vorstellung vom »Völkermord an den ArmenierInnen«, welcher »in den letzten dreißig Jahren eine universale Dimension gewann «, verkürzt und haltlos, weil jeder Völkermord/Genozid als solcher und an sich (sui generis et per se) immer schon genau diese »universale Dimension «per definitionem hat.

Dem Dinkessay folgt ein Lagebericht zur »Armenierdiskussion in der Türkei 2005 von Stefan Hibbeler (*1963), dem Herausgeber des wöchentlich erscheinenden deutschsprachigen Internetmagazins Istanbul Post ${ }^{36}$. Auf 15 Seiten betont Hibbeler, auch im Rückbezug auf Dink, die Bedeutsamkeit der Armenozidanerkennung als »Auseinandersetzung über die Fortsetzung der politischen Liberalisierung in der Türkei« und konzentriert sich dabei vor allem auf Ereignis und Bewertung (als Völkermord), sodann auf den Stil der öffentlichen Auseinandersetzungen und schließlich auch auf allgemeinere Aspekte türkischer Vertreibungspolitik "großer nichtmuslimischer Minderheiten « aus Anatolien. Hibbelers Beitrag enthält den Wortlaut der Erklärung der türkischen nationalen Versammlung (»Türkiye Büyük Millet Meclisi«) vom 13. April 2005, welche von »armenischen Vorwürfen« sprach und leider verdeutlichte, dass bisher noch alle (auch hier zusammenfassend dokumentierten) zivilgesellschaftlichen Hinweise und Anregungen von Staat und Politik der Türkischen Republik gleichermaßen ignoriert wurden.

\section{IV. »Staatliche Gewaltverbrechen« und Völkermord(verbinderung)}

Drei in den letzten Jahren erschienene Sammelbände beschäftigen sich mit Völkermord aus historischer Sicht und gehen damit, auch zur Perspektiverweiterung, über die hier bisher diskutierten Bücher zur Genozidpolitik hinaus. Dabei sind jeweils nicht alle, sondern nur einige akzentuierte Texte auch aus komparatistischer, also völkermordvergleichender Sicht von Interesse.

36 http://www.istanbulpost.net/Istanbul\%20Post/about.htm. 
Der erste Sammelband des StudienVerlags ist im besten Sinn ein didaktisch angelegter »reader «, der Völkermorde im vergangenen Jahrhundert als Staatsverbrechen, genauer als staatliche Gewaltverbrechen, auffasst ${ }^{37}$. Entgegen dem Reihentitel geht es weniger um »Kontroversen « als vielmehr um (Leit-)»Konzepte zur Definitionsmacht, Wahrnehmung (Perzeption) und Prävention genozidaler Handlungen «. Der Band ist klar strukturiert und logisch aufgebaut: Vorwort und Einleitung der Editoren folgen als Hauptabschnitte »Theoretische Überlegungen und Definitionsprobleme u und zum »Umgang mit Völkermord und staatlichen Gewaltverbrechen: Beispiele aus der internationalen Praxis « mit jeweils fünf Beiträgen. Der dritte Abschnitt »Materialien « ist in sich zweiteilig mit vier Texten zur Erinnerungsarbeit (u. a. aus Chile und dem ehemaligen Jugoslawien) und Hinweisen zu Literatur, Internetlinks sowie einem nützlichen Glossar.

Der Sammelband enthält vier Leitbeiträge von international bekannten Völkermordforschern: Yves Ternon, dessen Buch zum Armenozid als »Völkermord im 20. Jahrhundert « auch deutsch erschien ${ }^{38}$, erinnert an Völkermordprävention als (ausschließliche) Aufgabe der »internationalen Gemeinschaft « und an wissenschaftliche Versuche, über politiksoziologisch erkennbare empirische Entwicklungsstadien Karrierepfade von Völkermordgesellschaften zu erkennen, bevor jene Phasen und »Points of no return « erreicht sind, in denen Völkermordverhinderung kaum noch möglich ist. Einen neueren Versuch, über eine gezielte »internationale Kampagne zur Beendigung von Genoziden « wirksam zu werden, präsentiert in Form eines erweiterten achtstufigen Ablaufsmodells der US-amerikanische Politikberater Gregory Stanton ${ }^{39}$. Der deutsch-armenische Völkermordforscher Mihran Dabag entwickelt seine sozialpsychologische Sicht als »Perspektive strukturvergleichender Genozidforschung « und identifiziert dabei genozidale »Risikofaktoren « wie ein intergenerativ wirksames »Feindbild « im Zusammenhang mit einer (typischerweise rassistischen) Vernichtungsideologie zur »Mobilisierung großer Bevölkerungsgruppen«. Daran anschließend verweist Eric D. Weitz politikhistorisch im Anschluss an Hannah Arendt auf die massengesellschaftlichen Aspekte aller >modernen< Genozide als »gesellschaftliche Projekte« unter Einbezug der »gesamten Bevölkerung«. Die in den 1990 Jahren geschaffenen Haager Internationalen Gerichtstribunale wertet Weitz als Momente auf dem Weg zu einer »neuen internationalen Rechtsordnung «, um »Genozide Teil unserer Geschichte werden zu lassen, nicht unserer Gegenwart«. Eine historisch-komparatistische Sicht bringt, im nächsten Abschnitt, auch Falk Pingel (zum »Thema Völkermord als Gegenstand von Unterricht und Schulbuch«) ein, wenn er betont, »dass der Völkermord nicht mit Hitler begann

37 Verena Radkau; Eduard Fuchs; Thomas Lutz (Hg.), Genozide und staatliche Gewaltverbrechen im 20. Jahrbundert, Wien 2004 [= Konzepte und Kontroversen. Materialien für Unterricht und Wissenschaft in Geschichte - Geographie - Politische Bildung 3].

38 Yves Ternon, Der verbrecherische Staat. Völkermord im 20. Jabrhundert, Hamburg 1966.

39 Sein erstmals 1996 veröffentlichtes Ablaufmodell der "Seven Stages of Genocide“ ist nachzulesen unter http://www.genocidewatch.org/7stages.htm (abgerufen am 30.6.2008). 
und leider auch nicht mit ihm endet«. Dabei wünscht sich Pingel auch für den Schulunterricht, dass zwischen »Völkermord « und anderen »politisch motivierten Massenverbrechen « unterschieden wird, problematisiert die gerade in Schulbüchern anzutreffende Suggestiv-»Sprache der Bilder « und (an)erkennt auch hier Entwicklungsprozesse, wenn inzwischen auch (etwa am Beispiel Deutsch-Südwestafrikas) der »koloniale Genozid als Vorläufer des Holocaust « angesprochen oder »die Deportationen der Armenier erwähnt« werde. Auf den praktischen Gebrauchswert des Bandes zielt der dritte, von Eduard Fuchs bearbeitete Abschnitt mit seinen Literaturempfehlungen, Internetlinks und einem nützlichen kleinen Glossar von A-Z.

\section{Minderheitenpolitik, ethnische Homogenisierung und Vertreibung in Europa}

Der zweite Sammelband des StudienVerlags ist historisch ausgreifend auf Europa vom 16.-20. Jahrhundert ausgelegt $t^{40}$ und soll, so die Editoren in ihrer Einführung, »eine Mischung aus Überblicks-, Vergleichs- und Fallstudien mit unterschiedlichen räumlichen Schwerpunkten, Typen, Rechtsformen sowie Ursachen und Motivbündeln von Ausweisung, Abschiebung und Vertreibung « bieten. Für den genozidalen Zusammenhang sind dabei drei Texte anregend: der Überblick von Hans-Heinrich Nolte zum differenten Umgang "Zwischen Duldung und Vertreibung « mit ethnisch-religiösen Minoritäten seit dem 16. Jahrhundert, Hanns Haas' Übersicht zu Methoden staatlicher ethnischer Homogenisierungspolitik im 20. Jahrhundert und Fikret Adanirs Fallstudie zur osmanisch-türkischen Bevölkerungspolitik zwischen »Berliner Kongress « (1878) und der Gründung der Türkischen Republik (29.10.1923).

Noltes Beitrag einvernimmt Christentum, Judentum und Islam als die drei religiösen Hauptströmungen in Europa seit dem Dreißigjährigen Krieg und erinnert daran, dass die zentraleuropäischen Staaten und Territorien Spanien, Frankreich und Deutschland auch »durch die Exklusion von religiösen Minderheiten« gebildet wurden, während sich das insulare Großbritannien durch den Ausschluss »der Kelten der Randgebiete - der Walliser, Iren und Schotten«, konstituierte. Im Osmanischen Staat hingegen gab es diese Exklusion jahrhundertelang nicht: Muslimische Herrscher duldeten traditionell auch Angehörige anderer Religionen »wie Juden, Armenier und Orthodoxe, die Alltagskultur war durch Vielfalt auch der Religionen geprägt«. Es war die 'westliche Modernisierung< in der zweiten Hälfte des 19. Jahrhunderts, die das herkömmliche millet-System mit seiner begrenzten Sozialintegration erschütterte: »Die Reformen des 19. Jahrhunderts, in denen Prinzipien gleichen Rechts für alle Staatsbürger versprochen wurden [...], führten paradoxerweise nicht zu einem toleranteren Staat, sondern über die nationalistische Revolution, in welcher der Islam zu einem eher säkular konnotierten Bekenntnis zum Türkentum verwandelt wurde, zu schärferen Verfolgungen von Nichtmuslimen und

40 Sylvia Hahn, Andrea Komlosy, Ilse Reiter, Ausweisung - Abschiebung - Vertreibung in Europa 16. - 20. Jabrbundert, Innsbruck-Wien-Bozen: 2006268 p. [= Querschnitte: Einführungstexte zur Sozial-, Wirtschafts- und Kulturgeschichte 20]. 
schließlich zu Genoziden.«- Im Ausblick skizziert der Autor stichwortartig eine komplexe Typologie der Vertreibung und Vernichtung von Minderheiten in Europa während der letzten dreiundhalb Jahrhunderte. Diese dürfte die künftige komparatistisch-historische Forschung ebenso wie politiksoziologische Deutungen anregen.

Verschiedene Aspekte dieses Beitrags werden in zwei weiterführenden und materialreichen Aufsätzen aufgenommen und weitergeführt: Hanns Haas entfaltet ein Tableau von »ethnischer Homogenisierung unter Zwang « während und nach dem Ersten Weltkrieg, das den Bogen von freiwilliger »Assimilation « über »Zwangsassimilation « und "geregelte Zwangsemigration « bis zum Genozid als "physischer Vernichtung einer Volksgruppe« (so Haas' Umschreibung des Armenozids) spannt. Der von Haas angeregte intellektuelle Diskurs, der weder die welthistorische Bedeutung des Armenozids noch die »Deportationen im Stalinismus « und die Maßnahmen gegen Deutsche in Tschechien und Jugoslawien verschweigt und insofern aufklärend wirkt, versteht alle ethnischen Homogenisierungsversuche als demographische »Experimente im 20. Jahrhundert «, denen ein allgemeines Muster unterliegt: »Vertreibung beginnt mit der Zerstörung der Lebensgrundlagen; sie entzieht den Betroffenen alle staatsbürgerlichen Rechte und den Schutz des Staates; sie eliminiert die wirtschaftlichen, sozialen und kulturellen Einrichtungen der verfemten Gruppe. Alle Amtsträger werden entfernt, die politischen Organisationen verboten, die Schulen geschlossen, die Religionsgemeinschaften aufgelöst. Es folgen die Enteignung der Wirtschaftsbetriebe, Zwangsarbeit und Lagerverbringung."

Der dritte kontextual relevante Beitrag dieses Sammelbands ist als Fallstudie zur spätosmanisch-jungtürkischen Bevölkerungspolitik angelegt. Fikret Adanirs Text ist politiktheoretisch wichtig - auch wenn es der Autor leider unterlässt, diesen $\mathrm{Zu}$ sammenhang einzuholen, der sich etwa bei Hannah Arendt unter dem Stichwort »staatlich organisierter Verwaltungsmassenmord « findet und auf Montesquieus (später vom deutschen Salonmarxisten Karl August Wittvogel im Zusammenhang mit Besonderheiten der rasiatischen Produktionsweise < popularisiertes) Leitkonzept der >Orientalischen Despotie zurückgeht. Zutreffend beschreibt der Autor die Politik der »ethnischen Homogenisierung « einschließlich der »Turkifizierung der Ökonomie« als »Idee einer ethnisch-homogenen Bevölkerung neuzeitlich-europäischen Ursprungs «. Der Berliner Friedensvertrag (1878), dessen Artikel 61 sarmenische Reformen v vorsah, internationalisierte die »armenische Frage«. Im Ersten Weltkrieg beschloss die jungtürkische Führung des Osmanischen Reiches die Umsiedlung "praktisch aller armenischer Gemeinden « aus Anatolien, »ausgenommen Izmir [damals Smyrna], Istanbul [damals Konstantinopel] und Aleppo«-als »Deportation «, so Adanir, »ein schweres Verbrechen, wofür im heutigen Diskurs die Bezeichnung >Völkermord verwendet wird «. Nicht unerwähnt bleibt auch die »Katastrophe des Spätsommers 1922«, der »Smyrna Holocaust « nach Einnahme der ägäischen Handelsmetropole im September 1922 durch kemalistische Truppen als Voraussetzung der dann im Lausanner Vertrag vollzogenen Revisionspolitik als politische Letztvorgabe zur Gründung der sneuen Türkeir. - Als Historiker deutet Adanir »Bevölkerungsverschiebungen und Siedlungspolitik in Südosteuropa «18781923 als Ausdruck türkischer Politik der »ethnisch-kulturellen Homogenisierung « 
und verallgemeinert seine Fallstudienbefunde so: »Im Falle der Türkei erwies sich der Übergang vom Osmanischen Vielvölkerreich zum Nationalstaat als ein besonders langwieriger Prozess. Die Diskriminierung von Nichtmuslimen und nicht-türkischen muslimischen Ethnien setzte sich auch nach der Gründung der Republik im Jahre 1923 fort.«

\section{Menschenrechte und Menschenbilder}

Die Texte im letzten hier vorzustellenden Sammelband ${ }^{41}$ beleuchten zahlreiche weitere Facetten von Völkermord und Genozidpolitik, auch im antigenozidalen Sinn von Völkermordverhinderung. Statt hier die - gewiss ebenfalls lesenswerten alt-, media- und neuzeitlich-historischen Beiträge zu Menschenrechten und Judenbildern oder die Falldarstellungen einzelner Episoden und Personen der deutschen Zwischenkriegszeit zum außereuropäischen Rassismus oder den anregenden Essay zur menschenbildbezogenen »Körpergeschichte « [body history] von Maren Lorenz zu diskutieren, will ich mich dabei abschließend auf den allgemeinen Einleitungsbeitrag des Herausgebers und auf zwei weitere politikgeschichtliche Texte zum Menschenbild in Deutschland seit 1890 konzentrieren.

Burkhart Schmidts Text entwirft »Menschenrechte und Menschenbilder von der Antike bis zur Gegenwart«, ohne die Kategorie »Menschenrechte« durch Hypostasierung zur universalgeschichtlichen Leitkategorie überzustrapazieren und damit zugleich zu entwerten. Zentral und bis in unsere Zeit wirksam sind die Unabhängigkeitserklärung der dreizehn Staaten vom englischen Mutterland 1776 (»Declaration of Independence") und die Erklärung der Menschen- und Bürgerrechte von 1789/1793 ("Declaration des droits de l'homme et du citoyen ") mit den Hinweisen, dass alle Menschen (von Gott) gleich (geschaffen) und mit gewissen unveräußerlichen Rechten, darunter dem, das je eigne Glück zu suchen ("pursuit of happiness "), ausgestattet wurden. Zu Recht verweist Schmidt, der in seiner tour d'horizon auch an Ciceros dignitas als altrömische Vorstellung menschlicher Würde erinnert, auf unterschiedliche Vorstellungen: In der neuen Welt ging es schon besitzbezogen um "propriety, liberty, and freedom " (Richard Overton), während in der alten entsprechend »liberté, égalité, fraternité" für brüderliche Gleichheit gekämpft wurde. Das Ende 1848 von der deutschen Paulskirchenversammlung verabschiedete $\gg$ Gesetz über die Grundrechte des Deutschen Volkes « war »im wesentlichen auf die Abwehr staatlicher Willkür ausgerichtet« (1851 durch Bundestagsbeschluss wieder aufgehoben). Und zutreffend verweist Schmidt unter Verweis auf die Europäische Menschenrechtskonvention und den Europäischen Gerichtshof für Menschenrechte darauf, dass »Rechte haben« und »Recht erhalten« auch heute noch nicht dasselbe sind. - Im Abschnitt »Menschenbilder « erinnert der Herausgeber nicht nur an allgemeine Seiten unserer conditio bumana wie etwa Liebe (des Selbst und des Anderen), sondern auch an Selbst- und Fremdbilder, Auto- und Heterostereotypen,

41 Burghart Schmidt (Hg.), Menschenrechte und Menschenbilder von der Antike bis zur Gegenwart, Hamburg 2006 [= Geistes- und Kulturwissenschaftliche Studien 1]. 
schließlich Vorurteile zur Abgrenzung vom jeweils (vermeintlich) Anderen. Schließlich bekennt sich Schmidt als Zeithistoriker nicht nur zur Verantwortung als Zeitgenosse, sondern auch zu einem autoreflexiven »sozial- und kulturwissenschaftlichen Gedächtnis, dessen Elemente, Bilder und Begriffe immer wieder einer selbstkritischen Analyse unterzogen werden müssen, um die erforderliche Distanz zum wahren. Dies gilt auch für Fragen nach >Menschenbildern< und >Menschenrechten $\ll$.

Vierzig Jahre nach der ersten kritischen Gesamtschau von Edgar Hartwig, der später, 1968 und 1983, auch zwei Lexikonbeiträge zum 1891 als »Allgemeiner Deutscher Verband « gegründeten Alldeutschen Verband (ADV) folgten, fasst Rainer Hering seine Hamburger Dissertation (2003) zum ADV 1890-1939 unter dem Aspekt »Menschenbild des extremen Nationalismus in Deutschland «1890-1933 zusammen. Deutlich wird die vom ADV offensiv vertretene extrem-nationalistische und völkisch-antisemitische Propaganda einschließlich ihrer rassistischen Grundierung. Der ADV vertrat »zahlreiche Ideen, die später von Adolf Hitler (1889-1945) und der NSDAP aufgegriffen wurden «. Nach der militärischen Niederlage des Deutschen Reiches Ende 1918 blieb der ADV im Quellsumpf militanter deutschvölkischer Organisationen auch in der Weimarer Republik aktiv. Zusammen mit rabiater Judenfeindschaft propagierte er als »wesentlichen Programmpunkt« die »Errichtung einer völkischen Diktatur « und »stellte eine wesentliche organisatorische und ideologische Konstante der >Völkischen Bewegung in Deutschland vom Kaiserreich zum >Dritten Reich< dar.«

Bernd Jürgen Wendts Beitrag zum »Menschenbild des Nationalismus, seinen Wurzeln und Konsequenzen « unter dem Titel »Moderner Machbarkeitswahn« schließt hier bruchlos an. Ausgangpunkt des Autors, der vor zwanzig Jahren ein Standardwerk zur deutschen Außenpolitik veröffentlichte ${ }^{42}$, ist die genozidpolitisch relevante Operationalisierung von abstrakt-allgemeiner »Menschenwürde« auf das humane »Lebensrecht jedes Individuums «. Im ersten Aufsatzteil diskutiert Wendt den »Siegeszug « der gerade im deutschsprachigen Raum ausgeprägten »Radikalisierung durch den rassenhygienischen Diskurs « als eine historisch besonders Form allgemeiner »Pathologie der Moderne (Dirk Blasius). Dafür war Hitlers Menschenbild, »das von der hierarchisch abgestuften Wertigkeit nicht nur der Rassen, sondern auch der Individuen ausging, damit den europäischen Menschenwerten von Gleichheit, Freiheit und Brüderlichkeit eine strikte Absage erteilte«, ebenso folgerichtiger Ausdruck wie die Anmaßung, »unter Berufung auf ein Naturgesetz Bessere und Stärkere von Schlechteren und Schwächeren zu scheiden, erstere zu fördern, letztere zu unterdrücken«. Eine Grundvoraussetzung des keineswegs linearen und »unauflöslich in den Gang der Kriegsereignisse verwobenen Wegs nach Auschwitz ab 1941« war jedoch das »rassenhygienische Menschenbild, das mit der Machteroberung durch die Nationalsozialisten 1933 in den Rang einer Staatsdoktrin erhoben und zum treibenden Motor der Rassenpolitik wurde«.

42 Bernd Jürgen Wendt, Grossdeutschland. Aussenpolitik und Kriegsvorbereitungen des Hitler-Regimes, München 1987. 
Mit Stationen auf dem »Weg nach Auschwitz« beschäftigt sich der zweite Aufsatzteil. Und wenn Wendt »Kontinuitätslinien « vom Kaiserreich zum Nationalsozialismus betont, so auch die Zäsur, die der Autor in der »Machteroberung durch die Nationalsozialisten 1933« sieht: »Der 30. Januar 1933 bedeutete mit dem Übergang vom Rassismus zu einer staatlich legitimierten und institutionalisierten Rassenpolitik einen entscheidenden Einschnitt. Mit der Beseitigung des Rechtsstaates und der Menschenrechte in Deutschland, begleitet von einer folgenreichen Aushöhlung des ärztlichen Ethos, brachen alle Dämme auf dem Weg von der Ideologie zur Tat«. Am Ende dieser Entwicklung standen »Ärzte als 'politische Soldaten««, die sich »dem Prinzip >Heilen durch Töten verpflichtet fühlten«: Naziärzte ${ }^{43}$. Sie waren Teil der Völkermörderelite ("genocidal elite ") in Deutschland. Ohne ihre Handlungen wäre die damalige »Auslöschung >lebensunwerten Lebens« « nicht möglich gewesen. Und diese Völkermordpraxis vollzog sich, so Wendt, mit Auschwitz als Sackbahnhof, Massenmordfabrik und Endpunkt in diesen »fünf Stufen «: »Zwangssterilisation, Tötung (wirklich oder angeblich) kranker Kinder in Krankenhäusern, Tötung erwachsener Anstaltsinsassen durch Gas in medizinischen Tötungszentren (Euthanasie), Tötung (wirklich oder angeblich) kranker Insassen von Konzentrationslagern und schließlich die Massenmorde an den Juden.«

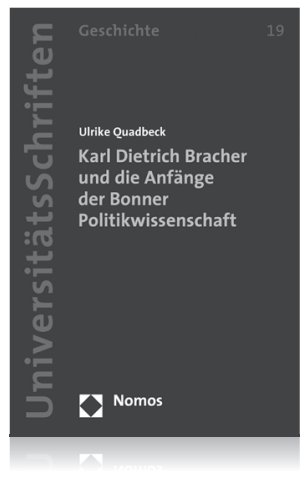

\section{Karl Dietrich Bracher und die Anfänge der Bonner Politikwissenschaft}

Von Dr. Ulrike Quadbeck

2008,436 S., brosch., 69, $-€$,

ISBN 978-3-8329-3740-9

(Nomos Universitätsschriften - Geschichte, Bd. 19)

Bitte bestellen Sie im Buchhandel oder

versandkostenfrei unter $>$ www.nomos-shop.de

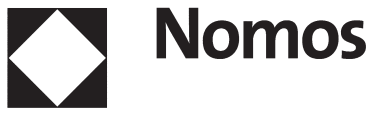

43 Vgl. Robert Jay Lifton, The Nazi Doctors. Medical Killing and the Psychology of Genocide, New York 1986. 